\title{
WHEN IS A FOURFOLD MASSEY PRODUCT DEFINED?
}

\author{
DANIEL C. ISAKSEN
}

(Communicated by Michael A. Mandell)

\begin{abstract}
We define a new invariant in the homology of a differential graded algebra. This invariant is the obstruction to defining a fourfold Massey product. It can be used to detect differential graded algebras that are not quasiisomorphic. We also make an explicit calculation in the cohomology of the Steenrod algebra.
\end{abstract}

\section{INTRODUCTION}

Massey products and Toda brackets are an essential tool for a detailed understanding of the cohomology of the Steenrod algebra and stable homotopy groups of spheres (see, for example, [7, 2], 1]). The standard references on Massey products, such as [4] and [6], typically assume that brackets are strictly defined, i.e., that the subbrackets have no indeterminacy. We have found in our own work on motivic stable homotopy groups [3] that strictly defined brackets are not always general enough.

This note addresses a subtlety with the definition of fourfold Massey products that arises when both of the threefold subbrackets have indeterminacy. We will define a new invariant (see Definition 3.1) of the homology of a differential graded algebra. Our main result (see Theorem 3.4) is that this invariant is the obstruction to defining a fourfold bracket whose threefold subbrackets contain zero.

We work with a differential graded algebra $A$ whose homology is $H$. We will assume that $A$ has characteristic 2 so that there are no signs to contend with. The reader who is interested in other characteristics can insert appropriate signs. For convenience, we suppress the grading of $A$. In general, $A$ need not be commutative. We have also avoided the full generality of matric Massey products, but the results carry over to that context as well.

The symbols $a_{i}$ always represent cycles, and the products $a_{i} a_{i+1}$ are always assumed to be boundaries. In other words, all threefold brackets are assumed to be defined. For any cycle $x$ in $A$, we write $\bar{x}$ for the element of $H$ that is represented by $x$.

\section{THE PROBLEM}

Let us first recall how to compute a fourfold Massey product $\left\langle\overline{a_{0}}, \overline{a_{1}}, \overline{a_{2}}, \overline{a_{3}}\right\rangle$. First choose elements $a_{01}, a_{12}$, and $a_{23}$ such that $d\left(a_{01}\right)=a_{0} a_{1}, d\left(a_{12}\right)=a_{1} a_{2}$, and $d\left(a_{23}\right)=a_{2} a_{3}$. Next, choose elements $a_{02}$ and $a_{13}$ such that $d\left(a_{02}\right)=a_{0} a_{12}+a_{01} a_{2}$

Received by the editors October 11, 2013.

2010 Mathematics Subject Classification. Primary 55S30, 55S10.

Key words and phrases. Massey product, cohomology of the Steenrod algebra.

The author was supported by NSF grant DMS-1202213.

(C)2014 American Mathematical Society Reverts to public domain 28 years from publication 
and $d\left(a_{13}\right)=a_{1} a_{23}+a_{12} a_{3}$. The bracket $\left\langle\overline{a_{0}}, \overline{a_{1}}, \overline{a_{2}}, \overline{a_{3}}\right\rangle$ is the subset of $H$ consisting of all elements of the form

$$
\overline{a_{0} a_{13}+a_{01} a_{23}+a_{02} a_{3}} \text {. }
$$

There is a subtlety that arises if $\left\langle\overline{a_{0}}, \overline{a_{1}}, \overline{a_{2}}\right\rangle$ or $\left\langle\overline{a_{1}}, \overline{a_{2}}, \overline{a_{3}}\right\rangle$ has indeterminacy. In this case, one must be careful to choose $a_{01}, a_{12}$, and $a_{23}$ in such a way that $a_{0} a_{12}+$ $a_{01} a_{2}$ and $a_{1} a_{23}+a_{12} a_{3}$ are boundaries. The well-known Lemma 2.1 addresses a simple case of this phenomenon.

Lemma 2.1. Suppose that both $\left\langle\overline{a_{0}}, \overline{a_{1}}, \overline{a_{2}}\right\rangle$ and $\left\langle\overline{a_{1}}, \overline{a_{2}}, \overline{a_{3}}\right\rangle$ contain zero, and at least one of the brackets is strictly zero. Then $\left\langle\overline{a_{0}}, \overline{a_{1}}, \overline{a_{2}}, \overline{a_{3}}\right\rangle$ is defined.

Proof. Suppose that $\left\langle\overline{a_{0}}, \overline{a_{1}}, \overline{a_{2}}\right\rangle$ is strictly zero. Choose $a_{12}$ and $a_{23}$ such that $a_{1} a_{23}+a_{12} a_{3}$ is a boundary. Then any choice of $a_{01}$ makes $a_{0} a_{12}+a_{01} a_{2}$ into a boundary, since $\left\langle\overline{a_{0}}, \overline{a_{1}}, \overline{a_{2}}\right\rangle$ is strictly zero.

The same argument applies when $\left\langle\overline{a_{1}}, \overline{a_{2}}, \overline{a_{3}}\right\rangle$ is strictly zero.

If both $\left\langle\overline{a_{0}}, \overline{a_{1}}, \overline{a_{2}}\right\rangle$ and $\left\langle\overline{a_{1}}, \overline{a_{2}}, \overline{a_{3}}\right\rangle$ have indeterminacies, it may be impossible to choose $a_{01}, a_{12}$, and $a_{23}$ such that both $a_{0} a_{12}+a_{01} a_{2}$ and $a_{1} a_{23}+a_{12} a_{3}$ are boundaries simultaneously. The problem is that there are two constraints on $a_{12}$, and it may not be possible to satisfy both constraints.

\section{Coindeterminacy}

Suppose that $\left\langle\overline{a_{0}}, \overline{a_{1}}, \overline{a_{2}}\right\rangle$ and $\left\langle\overline{a_{1}}, \overline{a_{2}}, \overline{a_{3}}\right\rangle$ both contain zero, but either possibly has non-zero indeterminacy.

Definition 3.1. The coindeterminacy of the brackets $\left\langle\overline{a_{0}}, \overline{a_{1}}, \overline{a_{2}}\right\rangle$ and $\left\langle\overline{a_{1}}, \overline{a_{2}}, \overline{a_{3}}\right\rangle$ is the subset of $H$ consisting of all elements of the form $\overline{x+y}$, where $x$ ranges over all elements of $A$ such that $d(x)=a_{1} a_{2}$ and $a_{0} x+z a_{2}$ is a boundary for some $z$ with $d(z)=a_{0} a_{1}$; and $y$ ranges over all elements of $A$ such that $d(y)=a_{1} a_{2}$ and $a_{1} w+y a_{3}$ is a boundary for some $w$ with $d(w)=a_{2} a_{3}$.

In other words, $x$ ranges over all possible choices of $a_{12}$ that can be used to construct zero in $\left\langle\overline{a_{0}}, \overline{a_{1}}, \overline{a_{2}}\right\rangle$. Similarly, $y$ ranges over all possible choices of $a_{12}$ that can be used to construct zero in $\left\langle\overline{a_{1}}, \overline{a_{2}}, \overline{a_{3}}\right\rangle$.

The careful reader can verify that the coindeterminacy is well defined in $H$, i.e.,

(1) if $x$ and $y$ satisfy the conditions of Definition 3.1, then $x+y$ is a cycle,

(2) if $x$ and $y$ satisfy the conditions of Definition 3.1 and $b$ is a boundary, then $x+b$ and $y$ satisfy the conditions of Definition 3.1

(3) if $a_{i}^{\prime}$ is homologous to $a_{i}$ for each $i$, then the coindeterminacy of $\left\langle\overline{a_{0}^{\prime}}, \overline{a_{1}^{\prime}}, \overline{a_{2}^{\prime}}\right\rangle$ and $\left\langle\overline{a_{1}^{\prime}}, \overline{a_{2}^{\prime}}, \overline{a_{3}^{\prime}}\right\rangle$ is the same as the coindeterminacy of $\left\langle\overline{a_{0}}, \overline{a_{1}}, \overline{a_{2}}\right\rangle$ and $\left\langle\overline{a_{1}}, \overline{a_{2}}, \overline{a_{3}}\right\rangle$.

Definition 3.2. Let $\bar{a}$ and $\bar{b}$ be elements of $H$. Then $(\bar{a} \backslash \backslash \bar{b})$ is the additive subgroup of $H$ consisting of all elements $\bar{x}$ such that $\overline{a x}=\bar{z} \bar{b}$ for some $\bar{z}$ in $H$, and $(\bar{a} / / \bar{b})$ is the additive subgroup of $H$ consisting of all elements $\bar{x}$ such that $\overline{a z}=\bar{x} \bar{b}$ for some $\bar{z}$ in $H$.

Lemma 3.3. The coindeterminacy of $\left\langle\overline{a_{0}}, \overline{a_{1}}, \overline{a_{2}}\right\rangle$ and $\left\langle\overline{a_{1}}, \overline{a_{2}}, \overline{a_{3}}\right\rangle$ is a coset with respect to $\left(\overline{a_{0}} \backslash \backslash \overline{a_{2}}\right)+\left(\overline{a_{1}} / / \overline{a_{3}}\right)$.

One possible name for $\left(\overline{a_{0}} \backslash \backslash \overline{a_{2}}\right)+\left(\overline{a_{1}} / / \overline{a_{3}}\right)$ is the "indeterminacy of the coindeterminacy". 
Proof. Let $x+y$ and $x^{\prime}+y^{\prime}$ represent elements of the coindeterminacy. We will consider $(x+y)+\left(x^{\prime}+y^{\prime}\right)=\left(x+x^{\prime}\right)+\left(y+y^{\prime}\right)$.

The element $x+x^{\prime}$ is a cycle. There exist elements $z$ and $z^{\prime}$ such that $a_{0} x+z a_{2}$ and $a_{0} x^{\prime}+z^{\prime} a_{2}$ are boundaries. Therefore, $a_{0}\left(x+x^{\prime}\right)$ is homologous to $\left(z+z^{\prime}\right) a_{2}$. This shows that $\overline{x+x^{\prime}}$ belongs to $\left(\overline{a_{0}} \backslash \backslash \overline{a_{2}}\right)$. Similarly, $\overline{y+y^{\prime}}$ belongs to $\left(\overline{a_{1}} / / \overline{a_{3}}\right)$.

On the other hand, let $\overline{x+y}$ be an element of the coindeterminacy, and let $\bar{c}$ be an element of $\left(\overline{a_{0}} \backslash \backslash \overline{a_{2}}\right)$. Choose a cycle $e$ such that $\overline{a_{0} c}=\overline{e a_{2}}$. There exists $z$ in $A$ such that $a_{0} x+z a_{2}$ is a boundary. Then $a_{0}(x+c)+(z+e) a_{2}$ is also a boundary. This shows that $\overline{(x+c)+y}$ also belongs to the coindeterminacy. Similarly, if $\bar{c}$ in an element of $\left(\overline{a_{1}} / / \overline{a_{3}}\right)$, then $\overline{x+(y+c)}$ also belongs to the coindeterminacy.

Theorem 3.4. Suppose that $\left\langle\overline{a_{0}}, \overline{a_{1}}, \overline{a_{2}}\right\rangle$ and $\left\langle\overline{a_{1}}, \overline{a_{2}}, \overline{a_{3}}\right\rangle$ both contain zero but possibly have non-zero indeterminacies. The fourfold bracket $\left\langle\overline{a_{0}}, \overline{a_{1}}, \overline{a_{2}}, \overline{a_{3}}\right\rangle$ is defined if and only if zero is contained in the coindeterminacy of $\left\langle\overline{a_{0}}, \overline{a_{1}}, \overline{a_{2}}\right\rangle$ and $\left\langle\overline{a_{1}}, \overline{a_{2}}, \overline{a_{3}}\right\rangle$.

In other words, the coindeterminacy is the obstruction to defining a fourfold bracket, given that both of its threefold subbrackets contain zero.

Proof. Suppose that $\left\langle\overline{a_{0}}, \overline{a_{1}}, \overline{a_{2}}, \overline{a_{3}}\right\rangle$ is defined. There are elements $a_{01}, a_{12}$, and $a_{23}$ such that $a_{0} a_{12}+a_{01} a_{2}$ and $a_{1} a_{23}+a_{12} a_{3}$ are boundaries. Then $\overline{0}=\overline{a_{12}+a_{12}}$ is an element of the coindeterminacy.

Suppose that zero belongs to the coindeterminacy. In the notation from Definition 3.1. we have $x=y$. Let $a_{01}, a_{12}$, and $a_{23}$ be $z, x$, and $w$ respectively. Then $a_{0} a_{12}+a_{01} a_{2}$ and $a_{1} a_{23}+a_{12} a_{3}$ are boundaries, so there exist choices for $a_{02}$ and $a_{13}$. This shows that $\left\langle\overline{a_{0}}, \overline{a_{1}}, \overline{a_{2}}, \overline{a_{3}}\right\rangle$ is defined.

\section{Differential graded algebras that are not QUASI-ISOMORPhic}

A quasi-isomorphism $\phi: A \rightarrow A^{\prime}$ induces isomorphisms of coindeterminacies in the homologies of $A$ and $A^{\prime}$. Therefore, coindeterminacies can be used to detect that two differential graded algebras are not quasi-isomorphic. We illustrate this with the following examples.

Example 4.1. Let $A$ be the differential graded algebra whose underlying algebra is a commutative polynomial algebra on the generators listed in the table. The differential on $A$ is the unique derivation that is defined on generators in the following table:

\begin{tabular}{ll}
$x$ & $d(x)$ \\
\hline$a_{0}$ & 0 \\
$a_{1}$ & 0 \\
$a_{2}$ & 0 \\
$a_{3}$ & 0 \\
$a_{01}$ & $a_{0} a_{1}$ \\
$a_{12}$ & $a_{1} a_{2}$ \\
$a_{23}$ & $a_{2} a_{3}$ \\
$c$ & 0 \\
$a_{02}$ & $a_{0} a_{12}+a_{01} a_{2}$ \\
$a_{13}$ & $a_{1} a_{23}+a_{12} a_{3}$ \\
\hline
\end{tabular}

Note that the indeterminacy of the subbracket $\left\langle\overline{a_{0}}, \overline{a_{1}}, \overline{a_{2}}\right\rangle$ contains $\overline{a_{0} c}$, and the indeterminacy of the subbracket $\left\langle\overline{a_{1}}, \overline{a_{2}}, \overline{a_{3}}\right\rangle$ contains $\overline{c a_{3}}$. Nevertheless, the fourfold bracket $\left\langle\overline{a_{0}}, \overline{a_{1}}, \overline{a_{2}}, \overline{a_{3}}\right\rangle$ is defined because the coindeterminacy contains zero. 
Example 4.2. Let $A^{\prime}$ be the differential graded algebra whose underlying algebra is the same as the underlying algebra of $A$. The differential on $A^{\prime}$ is the same as on $A$, except that $d\left(a_{13}\right)=a_{1} a_{23}+\left(a_{12}+c\right) a_{3}$.

The homologies of $A$ and of $A^{\prime}$ are quite similar. They are isomorphic as rings, and they share the same threefold Massey product structure. However, the bracket $\left\langle\overline{a_{0}}, \overline{a_{1}}, \overline{a_{2}}, \overline{a_{3}}\right\rangle$ is not defined in the homology of $A^{\prime}$ because the coindeterminacy is a non-zero coset of $\bar{c}=\overline{a_{12}+\left(a_{12}+c\right)}$.

Therefore, coindeterminacy detects that $A$ and $A^{\prime}$ are not quasi-isomorphic differential graded algebras.

\section{AN EXAMPLE}

In many cases of interest, it is not easy to compute coindeterminacy directly. We will provide an explicit example in the cohomology of the Steenrod algebra of a coindeterminacy that does not contain zero. However, we will obtain this result indirectly by showing that a fourfold Massey product cannot be defined for other reasons.

Lemma 5.1. The fourfold bracket $\left\langle h_{0}^{2}, h_{0}^{2} h_{3}, h_{0}^{2} h_{3}, h_{0}^{2}\right\rangle$ is not defined.

Proof. The threefold subbrackets $\left\langle h_{0}^{2}, h_{0}^{2} h_{3}, h_{0}^{2} h_{3}\right\rangle$ and $\left\langle h_{0}^{2} h_{3}, h_{0}^{2} h_{3}, h_{0}^{2}\right\rangle$ are both defined and equal to $\left\{0, h_{0}^{6} h_{4}\right\}$.

Note that $P^{2} h_{1}$ equals the bracket $\left\langle h_{0}^{2} h_{3}, h_{0}^{2}, P h_{1}\right\rangle$ with no indeterminacy, which equals $\left\langle h_{0}^{2}, h_{0}^{2} h_{3},\left\langle h_{0}^{2} h_{3}, h_{0}^{2}, h_{1}\right\rangle\right\rangle$ also with no indeterminacy. By a standard formal property of Massey products, this last expression would equal $\left\langle h_{0}^{2}, h_{0}^{2} h_{3}, h_{0}^{2} h_{3}, h_{0}^{2}\right\rangle h_{1}$, if the fourfold bracket were defined. However, $P^{2} h_{1}$ is not divisible by $h_{1}$, so the fourfold bracket cannot be defined.

Remark 5.2. The reader who is familiar with the structure of the cohomology of the Steenrod algebra will recognize that $\left\langle h_{0}^{2}, h_{0}^{2} h_{3}, h_{0}^{2} h_{3}, h_{0}^{2}\right\rangle$ is trying to be $P^{2}=v_{1}^{8}$. However, $P^{2}$ does not exist.

Corollary 5.3. The coindeterminacy of $\left\langle h_{0}^{2}, h_{0}^{2} h_{3}, h_{0}^{2} h_{3}\right\rangle$ and $\left\langle h_{0}^{2} h_{3}, h_{0}^{2} h_{3}, h_{0}^{2}\right\rangle$ is $\left\{h_{0}^{4} h_{4}, h_{0}^{4} h_{4}+h_{1} d_{0}\right\}$.

Proof. Lemma 3.3 says that the coindeterminacy is a coset of $\left\{0, h_{1} d_{0}\right\}$. Theorem 3.4 and Lemma 5.1 imply that the coindeterminacy does not contain zero. There is only one non-zero coset.

\section{NeXt STEPS}

We leave unanswered a number of interesting and accessible problems.

Problem 6.1. Extend these ideas to higher order Massey products.

Problem 6.2. Extend these results to fourfold (and higher) Toda brackets.

Problem 6.3. Establish a convergence theorem, in the spirit of [8], relating coindeterminacies in the cohomology of the Steenrod algebra to coindeterminacies in stable homotopy groups.

Problem 6.4. In the stable homotopy groups of spheres, find a fourfold bracket that is not defined, even though both of its threefold subbrackets contain zero, i.e., a coindeterminacy that does not contain zero. 
Because of Corollary [5.3, we expect that $\langle 4,4 \sigma, 4 \sigma\rangle$ and $\langle 4 \sigma, 4 \sigma, 4\rangle$ are Toda brackets whose coindeterminacy does not contain zero. In fact, the coindeterminacy ought to consist of the elements $k \rho_{15}$ and $k \rho_{15}+\eta \kappa$, where $k$ ranges over the values $2,10,18$, and 26 , and $\rho_{15}$ is the generator of the image of $J$ in the 15 -stem.

Problem 6.5. Reinterpret coindeterminacy in terms of the existence or nonexistence of certain 5-cell complexes.

Problem 6.6. Reinterpret coindeterminacy in terms of linkages of spheres in Euclidean space, in the spirit of [5].

\section{ACKNOWLEDGMENTS}

The author gratefully acknowledges helpful suggestions from Dan Dugger and Jim Stasheff.

\section{REFERENCES}

[1] M. G. Barratt, J. D. S. Jones, and M. E. Mahowald, Relations amongst Toda brackets and the Kervaire invariant in dimension 62, J. London Math. Soc. (2) 30 (1984), no. 3, 533-550, DOI 10.1112/jlms/s2-30.3.533. MR810962 (87g:55025)

[2] M. G. Barratt, M. E. Mahowald, and M. C. Tangora, Some differentials in the Adams spectral sequence. II, Topology 9 (1970), 309-316. MR0266215 (42 \#1122)

[3] D. C. Isaksen, Motivic stable stems, preprint, 2014.

[4] David Kraines, Massey higher products, Trans. Amer. Math. Soc. 124 (1966), 431-449. MR.0202136 (34 \#2010)

[5] W. S. Massey, Higher order linking numbers, J. Knot Theory Ramifications 7 (1998), no. 3, 393-414, DOI 10.1142/S0218216598000206. MR1625365 (99e:57016)

[6] J. Peter May, Matric Massey products, J. Algebra 12 (1969), 533-568. MR0238929 (39 \#289)

[7] Mark Mahowald and Martin Tangora, Some differentials in the Adams spectral sequence, Topology 6 (1967), 349-369. MR0214072 (35 \#4924)

[8] R. Michael F. Moss, Secondary compositions and the Adams spectral sequence, Math. Z. 115 (1970), 283-310. MR0266216(42 \#1123)

Department of Mathematics, Wayne State University, Detroit, Michigan 48202

E-mail address: isaksen@math.wayne.edu 